\title{
Neutron detection, the key to direction sensitive geoneutrino detectors
}

\section{F. D. Smit}

iThemba LABS

P O Box 722, Somerset West, 7129, South Africa

E-mail: smitetlabs.ac.za

\section{R. J. de Meijer}

Stichting EARTH

9321 XS 2, Peize, the Netherlands

and University of the Western Cape

Belleville 7535, South Africa

E-mail: demei jer@geoneutrino.nl

\section{R. W. Fearick}

University of Cape Town

Rondebosch 7701, South Africa

E-mail: fearick@science.uct.ac.za

\section{H. J. Wörtche}

Rijksuniversiteit Groningen

9774 AA, Groningen, the Netherlands

E-mail: wortcheakvi.nl 
The Earth AntineutRino TomograpHy (EARTH) project has as its eventual goal, a 3D map of the location of $U$ and $T h$ in the Earth. To achieve this the direction of low energy antineutrinos, known as geoneutrinos, emitted from these radioactive elements needs to be detected. If they are detected via inverse beta decay then the direction information is carried by the neutron emitted after capture by a proton, and how much of the direction information can be extracted depends crucially on the neutron detection. Simulations have shown a possible way of achieving this direction information.

International Workshop on Fast Neutron Detectors University of Cape Town, South Africa

April 3-6, 2006 


\section{Introduction}

It is accepted that radiogenic heat sources supply a significant proportion of the heat required to keep the outer core of the Earth liquid. However, hypotheses abound as to the distribution of these radiogenic heat sources. Detecting the antineutrinos, known as geoneutrinos, from the radioactive $\beta^{-}$-decay is one way of localising large concentrations of radioactivity in the interior of the Earth in general.

The long-term goal of the project Earth AntineutRino TomograpHy (EARTH) is a 3D image of the distribution of the radiogenic heat sources in the Earth. A more detailed description of the project, and its motivation, can be found in [1].

The design of most antineutrino detectors is dominated by the small reaction cross section for antineutrino detection. This dictates that one should have a large volume and a minimum of surface area to reduce the efficiency losses that occur at the surface. This leads to large monolithic detectors of which KamLAND [2] is a good example. KamLAND is spherical with a $13 \mathrm{~m}$ diameter. However, for the EARTH project, the determination of the direction of the geoneutrinos is paramount. Investigations on how this could be achieved, have been a determining factor on the possible design for the EARTH detector.

The reaction for detection, which because of the Q-value excludes geoneutrinos from $\mathrm{K}$, is the inverse $\beta$-decay.

$$
\bar{v}+p \rightarrow e^{+}+n-1.8 \mathrm{MeV}
$$

An antineutrino event is characterised by the delayed coincidence between the pulses generated by the positron and the captured neutron (double-pulse event). The large mass difference in the reaction products means that while the positron carries away most of the energy, the neutron carries away most of the momentum. Information about the direction of the incoming antineutrino is therefore contained in the direction of the neutron. Thus the method of neutron detection is crucial to extracting this information.

\section{Requirements for direction sensing}

In KamLAND, for example, the positron is detected via the annihilation gamma rays and the neutron is detected via the gamma ray emitted when the neutron is captured by a proton. A distance of up to two decimeters is observed between a detected annihilation gamma ray, and a detected neutron capture gamma ray, considerably larger than the few $\mathrm{cm}$ between the locations where the neutron is emitted and where it is captured (see Figure 1). Hence, with the gamma ray detection method, almost no information of the original antineutrino direction is preserved.

To preserve the direction information of the incoming geoneutrino as much as possible, one must firstly know where the antineutrino is captured and secondly where the neutron is captured. Charged particles with their small range are ideal for the task. For this reason a ${ }^{10} \mathrm{~B}-$ loaded scintillator is being tested as an antineutrino detector. In this scintillator the low energy 
positron can be detected as it is rapidly slowing down before annihilation, and the neutron can be detected by the recoiling ${ }^{7} \mathrm{Li}$ - and $\alpha$-particles emitted after the neutron is captured by the ${ }^{10} \mathrm{~B}$.

A further advantage of a ${ }^{10} \mathrm{~B}$-loaded scintillator is that it speeds up the capture of the neutron, because of the large capture cross section with $1 / \mathrm{v}$ dependence, where $\mathrm{v}$ is the velocity of the neutron. This feature reduces the average number of scatters the neutron undergoes before capture and thereby preserves more of the information of the original direction of the neutron.

Simulations of the interaction of an incoming neutrino in a scintillator with- and without ${ }^{10} \mathrm{~B}$-loading, using a code applying a Monte Carlo method, were made to test these insights. As a test of the code, the results shown in the paper by Beacom and Vogel [3] were reproduced. Results from this code can be seen in Figure 1.

Comparing the two data sets with and without B-loading, indicates the positive effect the ${ }^{10} \mathrm{~B}$-loading has in reducing the deviation of the neutron-capture point from the original neutrino direction. Secondly it can be seen that on average, from the point of emission, the neutron moves forward only a few centimeters before being captured.

Two pulses separated in space and time characterize the geoneutrino capture event. This
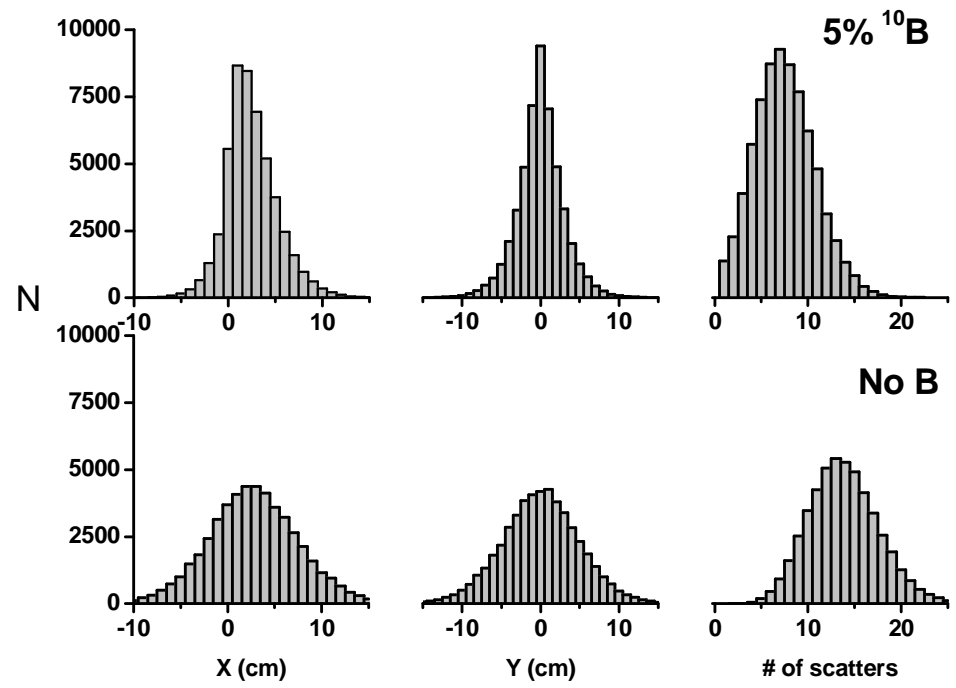

Figure.1. Both sets are results from 50000 Monte Carlo simulations of a neutrino with a reactor energy spectrum traveling along the $\mathrm{x}$-axis from negative to positive, and captured by a proton in a scintillator at $\mathrm{x}=0$ and $\mathrm{y}=0$. For the top set the scintillator was loaded with $5 \%{ }^{10} \mathrm{~B}$ and for the bottom set the scintillator had no B. Both sets show where the simulation stopped; for the top set when the neutron was captured, and for the bottom set when the neutron reached thermal energy. $\mathrm{Y}$ is the direction perpendicular to the antineutrino direction and the last graph plots the number of scatters in each simulation. 
feature can be used to facilitate direction sensing. If a scintillator is used which is long and thin, the detector will be efficient at detecting both pulses in the direction of the long axis $\left(0^{\circ}\right)$,
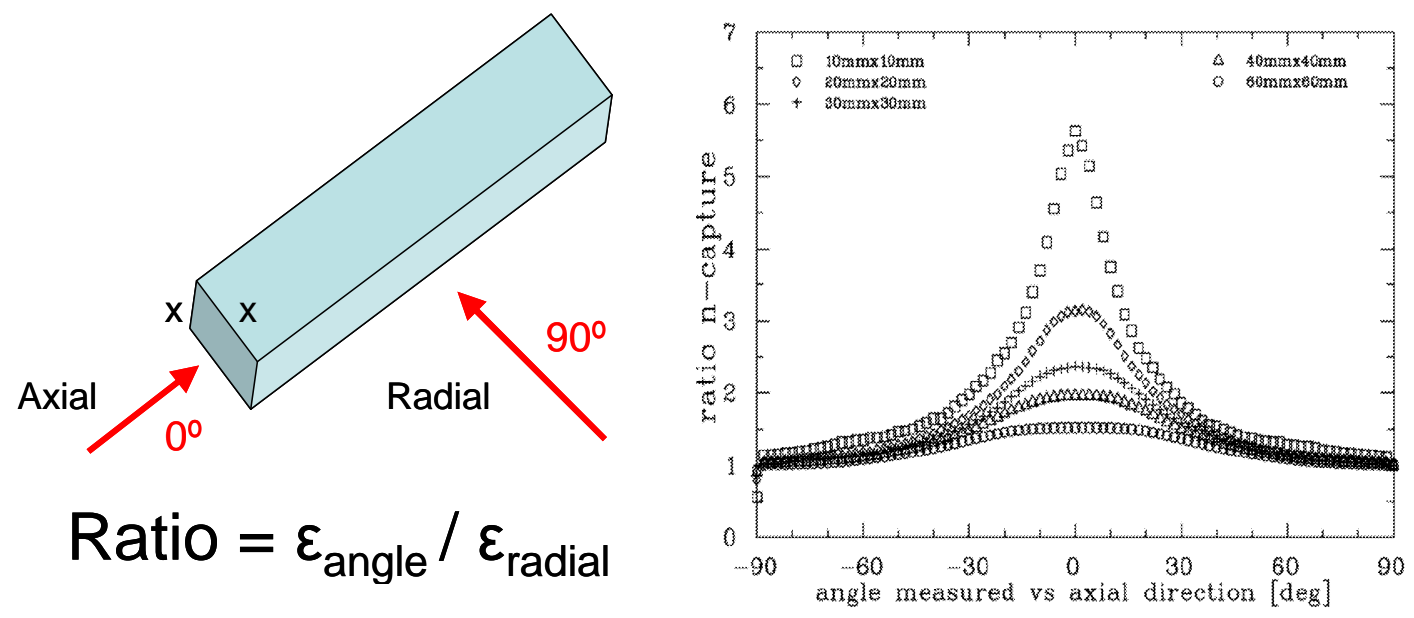

Figure $2 \mathrm{a}$ and $\mathrm{b}$. The influence of the scintillator dimensions on the probability of an antineutrino event being detected in a long thin scintilator for a antineutrino incoming at a particular angle to the scintillator. The yellow data is for a dimension of $60 \mathrm{~mm} \times 60 \mathrm{~mm}$. From this it can be seen that angular sensitivity is at the cost of efficiency and vice versa. See text for detail.

but not efficient at detecting both pulses in the radial direction $\left(90^{\circ}\right)$ as shown in Figure $2 \mathrm{~b}$. In the latter case many of the neutrons will escape the scintillator before being captured, with the result that no double pulse will be seen in the scintillator and the event will not be identified as an antineutrino.Therefore the efficiency of the detector for detecting antineutrinos entering from an angle other than axially is a function of the dimension $\mathrm{x}$ indicated in Figure $2 \mathrm{a}$ as well as the incident angle. In Figure $2 b$ the ratio is plotted of efficiency for detecting the neutron if the antineutrino entered axially divided by the efficiency of the detector for detecting the neutron if the antineutrino enters at some other angle. Also shown is how this ratio changes with the dimensions in the direction perpendicular to the long axis.

This result implies that direction sensitive geoneutrino detection will require highly modular detector systems, consisting of sets of modules. Each module comprises a number of detector units with their long axis aligned with a specific direction. Several of these sets will be required, each pointing in a particular direction. By bundling detector units into modules, one allows for detection of neutrons and positrons in adjacent detectors, resulting in more efficient use of scarce antineutrino events.

The overall volume of the detector will still have to be large as the absolute efficiency of detecting geoneutrinos is small. However, using direction sensitive antineutrino detecting as the prime objective leads to a detector, which looks completely different from the huge monolithic detectors one has become accustomed to.

In parallel to the computer simulations, experimental tests simulating double pulse events in ${ }^{10} \mathrm{~B}$-loaded scintillators have also been carried out. $\mathrm{A}{ }^{252} \mathrm{Cf}$ neutron source and a small 
$3.8 \mathrm{~cm}$ diameter and $2.5 \mathrm{~cm}$ thick ${ }^{10} \mathrm{~B}$-loaded liquid scintillator was used for this purpose. The first pulse of the double pulse is produced by the recoil proton from the neutron scattering and the second pulse is produced by the stopping of the ${ }^{7} \mathrm{Li}$ and $\alpha$-particles emitted after the neutron is captured by a ${ }^{10} \mathrm{~B}$. These pulses were recorded via an 8 bit, $1.25 \mathrm{Gsamples} / \mathrm{sec}, 100 \mathrm{MHz}$ bandwidth Tektronix oscilloscope. These data were read out via a LABVIEW program and stored on disc as ASCII data. Examples of these double pulses can be seen in Figure 3. The difference in pulse shape and size between pulses produced by neutrons and gamma rays is clear. Such differences can be used to significantly reduce background due to accidental coincidences in the geoneutrino detectors. This difference, together with the relatively small pulse (due to quenching) from the capture of a neutron, are further reasons why a modular detector system is required, with each module made up of a very large number of small scintillator units.

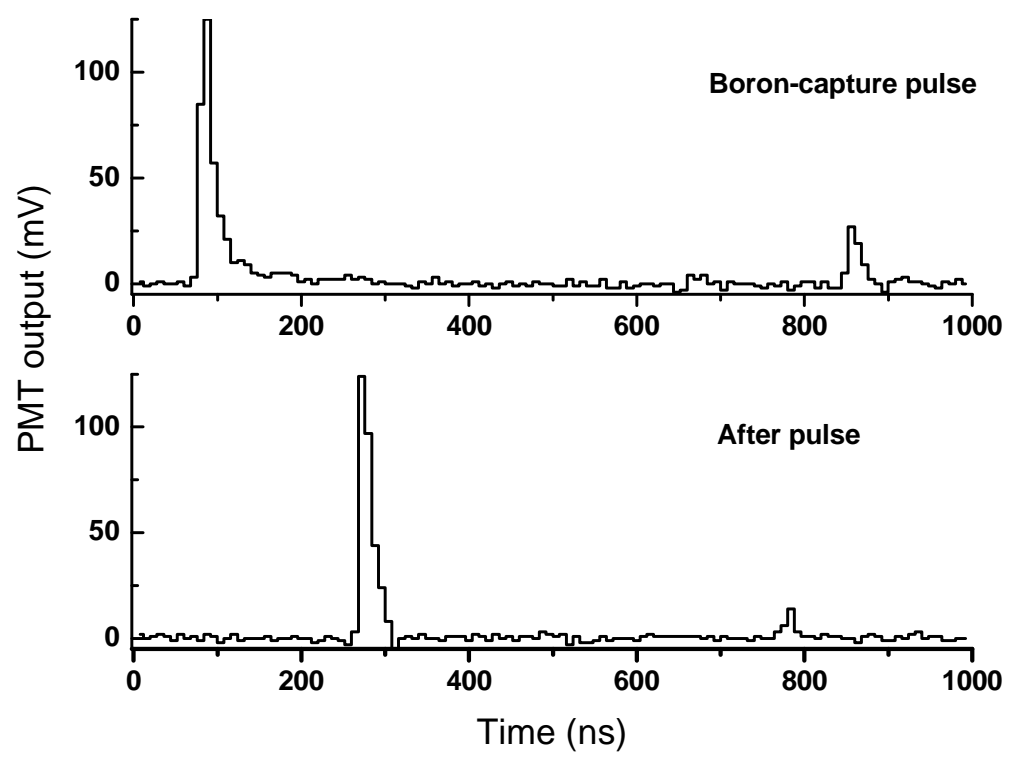

Figure 3. The top trace is one produced by a neutron scattering off a proton followed by the neutron then being captured by ${ }^{10} \mathrm{~B}$ and the emitted ${ }^{7} \mathrm{Li}$ and $\alpha$-particles being detected and producing the second pulse of the top trace. The bottom trace is an accidental double pulse where the first pulse of the trace is produced by a detected gamma ray while the second pulse in the trace is produced by an afterpulse in the photomultiplier. After pulses are small and always arrive at a fixed time relative to the first pulse

\section{Conclusion}

Calculations indicate that direction sensitive geoneutrino detection is possible. However, a highly modular detector system presents many challenges, mostly technological in nature, and largely different from those faced by the traditionally shaped (monolithic) antineutrino detectors. Technology always improves with time, and challenges will be resolved 
in steps. More complete calculations simulating the antineutrino interaction and the detection of the subsequent reaction products are underway. More comprehensive scintillator tests are also planned.

\section{Acknowledgment}

The authors are grateful to F D Brooks for the help and discussions on this work.

\section{References}

[1] R. J. de Meijer et al. Towards Earth Antineutrino Tomography (EARTH) Proceedings of Neutrino Sciences 2005 Workshop Hawaii, Dec. 2005 Earth, Moon and Planets in press.

[2] K. Eguchi et al. First results from KamLAND: Evidence for Reactor Antineutrino Disappearance Phys. Rev. Lett., 2003, 90, 021802.

[3] J. F. Beacom and P. Vogel Angular distribution of neutron inverse beta decay, $\bar{v}_{e_{-}}+p \rightarrow e^{+}+n$ Phys. Rev. D, 1999, 60, 053003 